\title{
Avaliação clínica do tratamento cirúrgico das fraturas do terço médio da clavícula em um hospital do sul de Santa Catarina*
}

\section{Clinical Evaluation of the Surgical Treatment of Midshaft Clavicle Fractures at a Hospital in the South of Santa Catarina}

\author{
Fernando H.S. Hehn ${ }^{1}$ Paola S.G. Bonavides ${ }^{1}$ \\ Martins Back Neto ${ }^{2}$ Willian N. Stipp ${ }^{2}$

\footnotetext{
${ }^{1}$ Faculdade de Medicina, Universidade do Sul de Santa Catarina (Unisul), Tubarão, SC, Brasil

2 Departamento de Ortopedia e Traumatologia, Hospital Nossa Senhora da Conceição, Tubarão, SC, Brasil
}

Aloir N. Oliveira Júnior ${ }^{1}$ Helena C.G. Silva ${ }^{1,2}$

Endereço para correspondência Fernando H.S. Hehn, MD, Rua Simeão Esmeraldino de Menezes 138, apto. 202/2, Dehon, Tubarão, SC, Brasil, CEP: 88704-090 (e-mail: sauerh@gmail.com).

Rev Bras Ortop 2020;55(1):100-105.

\section{Resumo \\ Palavras-chave \\ - clavícula/cirurgia \\ - resultado do tratamento \\ - fraturas ósseas}

Abstract
Objetivo Avaliar o resultado do tratamento cirúrgico de fraturas do terço médio da clavícula.

Métodos Estudo transversal retrospectivo, em que foram avaliados 36 pacientes que sofreram fratura do terço médio da clavícula, que foram tratados cirurgicamente no período de janeiro de 2012 a fevereiro de 2017. Eles foram avaliados quanto aos tipos de fratura, idade, tabagismo, material de síntese, e escores de Constant-Murley e Modified-University of California at Los Angeles Shoulder Rating Scale (UCLA-M).

Resultados As médias dos escores de Constant-Murley e UCLA-M foram de 91,59 e 31,29 , respectivamente. A idade média foi de 37,62 anos, e apresentou relação estatística com o tipo de síntese $(p<0,05)$, mas o material de síntese não apresentou significância com a melhora de pontuação dos escores funcionais.

Conclusão O tratamento cirúrgico ocasiona bons resultados funcionais após a fratura diafisária de clavícula, independente do traço da fratura, com baixa taxa de pseudoartrose.

Objective To evaluate the results of the surgical treatment of fractures of the middle third of the clavicle.

Methods A retrospective cross-sectional study, in which 36 patients who suffered fractures of the middle third of the clavicle and who were surgically treated from January 2012 to February 2017 were evaluated. They were evaluated for type of

\footnotetext{
Trabalho desenvolvido no Ambulatório de Ortopedia e Traumatologia, Hospital Nossa Senhora da Conceição, Tubarão, SC, Brasil.
}

recebido

27 de Março de 2018

aceito

08 de Agosto de 2018
DOI https://doi.org/

10.1055/s-0039-1697013. ISSN 0102-3616.
Copyright $\odot 2020$ by Sociedade Brasileira License terms de Ortopedia e Traumatologia. Published by Thieme Revinter Publicações Ltda, Rio de Janeiro, Brazil 


\section{Keywords}

- clavicle/surgery

- treatment outcome

- fractures, bone fracture, age, smoking, osteosynthesis material, and Constant-Murley and ModifiedUniversity of California at Los Angeles Shoulder Rating Scale (UCLA-M) scores.

Results The mean Constant-Murley and UCLA-M scores were 91.59 and 31.29 respectively. The mean age was 37.62 years, and it was statistically related to the type of osteosynthesis $(p<0.05)$, but the osteosynthesis material did not show significance with the improvement in the rates of the functional scores.

Conclusion The surgical treatment provides good functional results after diaphyseal fractures of the clavicle, regardless of the line of the fracture, with a low rate of pseudarthrosis.

\section{Introdução}

As fraturas de clavícula correspondem por 2,6 a 10\% de todas as fraturas em adultos, e representam até $44 \%$ das fraturas da cintura escapular, sendo a localização mais comum o terço médio. ${ }^{1,2}$ Elas são três vezes mais comuns em homens, e apresentam dois picos: em homens menores de 25 anos, e em mulheres maiores de 60 anos. ${ }^{3,4}$ As principais causas são trauma direto, queda da própria altura, e acidentes automobilísticos, sendo estes últimos a causa mais comum. ${ }^{5}$

Historicamente, as fraturas eram tratadas de forma conservadora, com tipoia ou bandagem em oito, independente do desvio delas. Tal conduta foi guiada por dois estudos dos anos 1960 que afirmavam que, desse modo, a taxa de pseudoartrose era menor do que $1 \%$, sendo o tratamento cirúrgico considerado somente se a fratura fosse exposta, houvesse risco iminente de lesão de pele causada pelo fragmento ósseo, ombro flutuante, ou injúria neurovascular. ${ }^{6,7}$

Entretanto, evidências recentes colocam em dúvida se a maioria dessas fraturas deveria ser tratada de maneira conservadora, visto que novos dados demonstram que a opção não cirúrgica ocasiona mais complicações do que havia sido publicado. Ademais, o tratamento conservador pode resultar em uma aparência não estética, devido à possibilidade do encurtamento da clavícula e da formação de um calo ósseo exuberante, situações que a cirurgia pode evitar. ${ }^{8}$

O tratamento cirúrgico acarreta melhores resultados funcionais no curto prazo, menor tempo de consolidação, e um retorno mais rápido ao trabalho. ${ }^{2,9}$ Há três opções principais para o manejo cirúrgico: placa com parafusos, fixação intramedular, e fixação externa; porém, a fixação por placa é a técnica padrão para essa abordagem. ${ }^{10}$ Existem várias opções de síntese utilizando placas, como a placa de compressão dinâmica (PCD), a placa de reconstrução, e as placas bloqueadas. ${ }^{11}$ Contudo, tal método não é isento de complicações, entre elas a infecção da ferida operatória, a cicatriz hipertrófica, a proeminência do material de síntese, e a necessidade de uma reoperação para retirada do material. $^{8,12}$

Portanto, devido não apenas à alta incidência das fraturas de clavícula, e ao impacto causado pelo afastamento das atividades sociais e laborais, como também, principalmente, pela falta de unanimidade quanto ao melhor tratamento, propõe-se realizar essa avaliação do resultado do tratamento cirúrgico das fraturas diafisárias da clavícula a fim de acrescentar informações aos conhecimentos existentes na literatura.

\section{Materiais e Métodos}

Este estudo foi realizado no Ambulatório de Ortopedia do Hospital Nossa Senhora da Conceição (HNSC) de Tubarão-SC, após a aprovação pelo Comitê de Ética em Pesquisa da Universidade do Sul de Santa Catarina (Unisul), seguindo as diretrizes e normas regulamentadoras da pesquisa científica, sob a resolução CNS 466/12, e aprovado sob o registro CAAE 62460816.3.0000.5369.

Trata-se de um estudo observacional de caráter transversal, no qual foram incluídos os pacientes que sofreram fratura do terço médio da clavícula, que foram tratados cirurgicamente no mesmo serviço e pelo mesmo cirurgião, no período de janeiro de 2012 a fevereiro de 2017, e que assinaram o termo de consentimento livre e esclarecido (TCLE). Foram excluídos do estudo os pacientes que não retornaram ao ambulatório e que já tinham doença, sequelas neurológicas ou cirurgia prévia no membro acometido. A população total do estudo foi composta por 55 pacientes.

Os pacientes foram submetidos a três questionários: o escore de Constant-Murley (CM) e o escore Modified-University of California at Los Angeles Shoulder Rating Scale (UCLA-M,), ambos validados para a realidade brasileira, ${ }^{13,14}$ e um questionário de informações gerais, como idade, sexo, mecanismo do trauma, lado da fratura, traço da fratura, tipo de síntese, tempo da fratura, complicações, e tabagismo.

Os dados foram digitados no programa Excel (Microsoft, Redmond, WA, EUA), versão 14.0.0, e seu processamento e análise foram realizados com o programa Statistical Package for the Social Sciences (SPSS, IBM Corp., Armonk, NY, EUA), versão 20.0. Foi utilizada análise descritiva - média e desvio padrão -, variáveis numéricas, e frequência para as variáveis categóricas. Testes estatísticos: qui-quadrado para variáveis categóricas e teste $t$ de Student, e análise de variância (ANOVA, em inglês) para as variáveis numéricas, de acordo com a necessidade. 0 nível de significância adotado foi de $5 \%$.

\section{Resultados}

A população foi composta de 36 dos 55 pacientes estimados que sofreram fratura do terço médio da clavícula no HNSC. Dos 36 pacientes que retornaram para a avaliação clínica, 2 


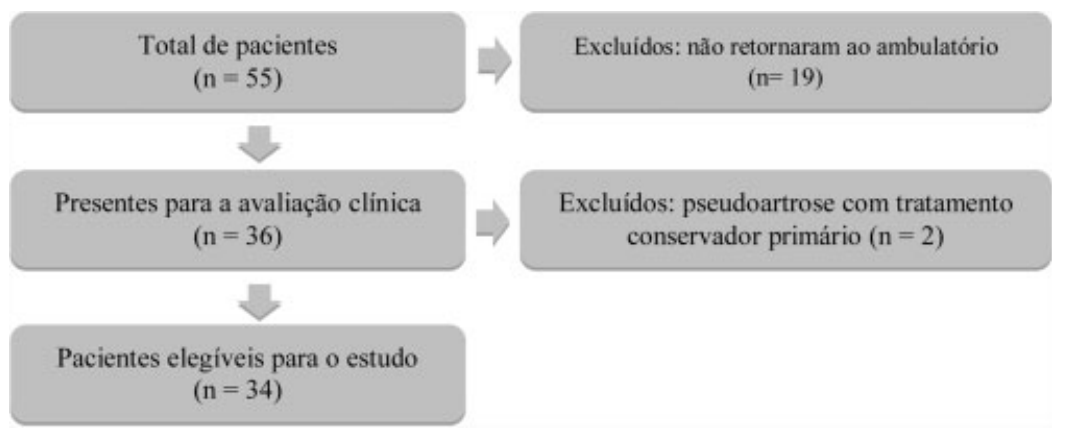

Figura 1 Fluxograma da relação amostral dos pacientes, desde o recrutamento até a avaliação para o estudo.

casos foram excluídos do estudo, pois foram primariamente tratados de maneira conservadora, mas evoluíram com pseudoartrose, e, então foram abordados cirurgicamente devido a esta complicação. Os outros 19 pacientes perderam seguimento ambulatorial, e não foi possível entrar em contato para solicitar o retorno deles. Tais informações estão descritas na - Figura 1.

Na amostra, observou-se predomínio do sexo masculino, faixa etária dos 21 aos 30 anos, maioria de não fumantes, cujo traço de fratura foi cominutivo, e a osteossíntese foi com PCD mais parafusos. A idade média dos pacientes foi de $37,62 \pm 13,49$ anos, sendo a mínima de 18 e a máxima de 63 anos. Os demais dados sobre o perfil dos pacientes que sofreram fratura do terço médio da clavícula em Tubarão-SC estão descritos na - Tabela 1.

Na - Tabela 2, estão descritos a média e os valores máximos e mínimos dos escores CM e UCLA-M. Ressalta-se que, quando aplicado o teste de correlação de Spearman, observou-se uma forte correlação ( $p=0,000$; intervalo de confiança de 95\% [IC95\%] -0,875--0,468), ou seja, os testes estão em consonância.

Na - Tabela 3, é possível observar a avaliação de ambos os escores segundo Boehm. ${ }^{15} \mathrm{O}$ valor percentual apresentado na tabela é relativo ao total de indivíduos pertinentes a cada categoria analisada. Pode-se observar que a maioria dos indivíduos teve resultados excelentes nos dois escores. Quando aplicado o teste $t$ de Student e comparada a média de escore CM entre os sexos, não houve significância estatística, mas o sexo masculino obteve uma maior pontuação, tendo uma média de escore CM de 92,15, e o sexo feminino, uma média de escore CM de 89,43 ( $p=0,525$; IC95\%: -9,1-14,5).

Em relação à idade e ao tipo de síntese, quando aplicado o Teste $t$ de Student, há uma tendência de utilizar a PCD, considerando o número absoluto, ou seja, dos 34 pacientes, 23 utilizaram PCD, com uma média de idade de 34,04 anos, e a placa de reconstrução teve uma média de escore $C M$ de 45,09 anos. Esta diferença de idade entre os tipos de síntese não foi significativa dada a limitação do número de sujeitos da pesquisa ( $p=0,116$; IC95\% -20,4--1,6).

O tipo de síntese não apresentou associação quando comparado com os escores, considerado o tamanho da amostra. O escore CM teve uma média, com DCP, de 91,91, e, com a placa de reconstrução, de 90,91 ( $p=0,483$; IC95\% -9,2-11,2). Com o escore UCLA-M, a média foi de 31,22 e 31,45 , com as placas DCP e de reconstrução, respectivamente
( $p=0,502$; IC95\%: -4,1-3,6). A análise de significância estatística das variáveis do estudo está descrita na - Tabela 4.

Observou-se também que não houve diferença estatística quando feitas as comparações entre tipo de traço de fratura, tabagismo, complicações e escores CM e UCLA-M.

\section{Discussão}

A melhor abordagem do tratamento das fraturas do terço médio da clavícula ainda está em discussão na literatura. Tenta-se com ela melhorar o prognóstico do paciente, para que ele possa retornar de forma plena às funções exercidas anteriormente à fratura. Recentemente, estudos têm publicado resultados que favorecem a opção cirúrgica, pois ela reduz a incidência de pseudoartrose, consolidação viciosa, e melhora a função do ombro. A osteossíntese mais utilizada é a fixação por placa mais parafusos, superiormente à clavícula. Logo, as principais complicações desta técnica estão relacionadas ao material cirúrgico. 1,2,10,16

0 estudo de Asadollahi et al, $^{17}$ em conformidade com nossa pesquisa, observou uma prevalência maior de pacientes do sexo masculino quando comparado ao sexo feminino, além de uma média de idade de 39,1 anos. Napora et $\mathrm{al}^{3} \mathrm{e}$ Devji et al $^{18}$ relataram uma média de idade entre 26,5 e 44,2 anos, assim como um maior predomínio de homens, variando de 53 a $91 \%$ dos casos dos estudos.

Os acidentes de trânsito (ATs) corresponderam ao principal mecanismo do trauma (91,2\%), seguido por queda da própria altura (5,9\%). Esse predomínio dos ATs está acima dos dados encontrados na literatura. Kihlström et al $^{19}$ classificaram as causas de 2,422 fraturas diafisárias, e quedas em geral corresponderam a $49 \%$ delas, e os ATs consistiram em 41,5\%. Ao contrário, Napora et al $^{3}$ encontraram $58 \%$ de casos por ATs e $34 \%$ por quedas em geral. Em vista disso, percebe-se que as causas das fraturas variam conforme o tipo de população dos trabalhos.

No presente estudo, não houve associação entre tabagismo e pior resultado funcional ou maiores complicações. De acordo com Napora et $\mathrm{al}^{3}$ e Robinson et al, ${ }^{20}$ o tabagismo acarreta piores escores funcionais do ombro, uma vez que tal hábito está relacionado a pior consolidação óssea; contudo, nesses pacientes não houve diferença clínica entre os tratamentos cirúrgico ou conservador. Murray et $\mathrm{al}^{21}$ e Ban et $\mathrm{al}^{22}$ descreveram que há um risco quatro vezes maior de pseudoartrose em fumantes. Inclusive, orientam a cessação do 
Tabela 1 Perfil dos pacientes que sofreram fratura do terço médio da clavícula em Tubarão-SC e que foram tratados cirurgicamente, no Hospital Nossa Senhora da Conceição, no período do estudo

\begin{tabular}{|c|c|c|}
\hline Variável & $\mathrm{N}$ & $\%$ \\
\hline \multicolumn{3}{|l|}{ Sexo } \\
\hline Masculino & 27 & 79,4 \\
\hline Feminino & 7 & 20,6 \\
\hline \multicolumn{3}{|l|}{ Faixa etária } \\
\hline Até 20 anos & 2 & 5,9 \\
\hline De 21 a 30 anos & 12 & 35,3 \\
\hline De 31 a 40 anos & 6 & 17,6 \\
\hline De 41 a 50 anos & 6 & 17,6 \\
\hline De 51 a 60 anos & 5 & 14,7 \\
\hline Maior do que 60 anos & 3 & 8,8 \\
\hline \multicolumn{3}{|l|}{ Tabagismo } \\
\hline Sim & 10 & 29,6 \\
\hline Não & 24 & 70,6 \\
\hline \multicolumn{3}{|l|}{ Tempo de fratura } \\
\hline Até 12 meses & 7 & 20,5 \\
\hline De 12 a 24 meses & 12 & 35,2 \\
\hline De 24 a 36 meses & 3 & 8,7 \\
\hline De 36 a 48 meses & 6 & 17,4 \\
\hline Maior do que 48 meses & 4 & 11,7 \\
\hline \multicolumn{3}{|l|}{ Traço da fratura } \\
\hline Simples & 16 & 47,1 \\
\hline Cominutivo & 18 & 52,9 \\
\hline \multicolumn{3}{|l|}{ Lado afetado } \\
\hline Direito & 21 & 61,8 \\
\hline Esquerdo & 13 & 38,2 \\
\hline \multicolumn{3}{|l|}{ Tipo de síntese } \\
\hline Placa de compressão dinâmica & 23 & 67,6 \\
\hline Placa de reconstrução & 11 & 32,4 \\
\hline \multicolumn{3}{|l|}{ Mecanismo do trauma } \\
\hline Queda da própria altura & 2 & 5,9 \\
\hline Acidente automobilístico & 31 & 91,2 \\
\hline Outro (esporte) & 1 & 2,9 \\
\hline Acidente Automobilístico & 31 & 91,2 \\
\hline \multicolumn{3}{|l|}{ Complicações } \\
\hline Pseudoartrose & 1 & 2,9 \\
\hline Placa saliente & 15 & 44,1 \\
\hline Parestesia pericicatriz & 7 & 20,6 \\
\hline Não & 11 & 32,4 \\
\hline
\end{tabular}

tabagismo como parte do tratamento, seja cirúrgico ou conservador.

Acerca do tipo do traço da fratura, houve predomínio do tipo cominutivo (52,9\%). Robinson et $\mathrm{al}^{20}$ também encon-
Tabela 2 Média, máximas e mínimas dos escores funcionais de Constant-Murley e Modified-University of California at Los Angeles Shoulder Rating Scale (UCLA-M), dos pacientes do estudo

\begin{tabular}{|l|l|l|l|}
\hline Escores & Média & Máximo & Mínimo \\
\hline Constant-Murley & 91,59 & 100 & 48 \\
\hline $\begin{array}{l}\text { Modified-University of } \\
\text { California at Los Angeles } \\
\text { Shoulder Rating Scale } \\
\text { (UCLA-M) }\end{array}$ & 31,29 & 35 & 14 \\
\hline
\end{tabular}

(Continued)

Tabela 3 Estratificação dos resultados dos escores funcionais de Constant-Murley e de Modified-University of California at Los Angeles Shoulder Rating Scale (UCLA-M), dos pacientes do estudo

\begin{tabular}{|l|l|l|}
\hline Avaliação dos escores & N & $\%$ \\
\hline Constant-Murley & & \\
\hline Excelente & 23 & 67,65 \\
\hline Bom & 6 & 17,65 \\
\hline Satisfatório & 2 & 5,88 \\
\hline Adequado & 1 & 2,94 \\
\hline Ruim & 2 & 5,88 \\
\hline $\begin{array}{l}\text { Modified-University of } \\
\text { California at Los Angeles } \\
\text { Shoulder Rating Scale } \\
\text { (UCLA-M) }\end{array}$ & 16 & 47,1 \\
\hline Excelente & 11 & 32,4 \\
\hline Bom & 5 & 14,7 \\
\hline Razoável & 2 & 5,9 \\
\hline Ruim & 16 & \\
\hline
\end{tabular}

Tabela 4 Análise de significância estatística das variáveis do estudo

\begin{tabular}{|l|l|l|}
\hline Variáveis & $p$ & $\begin{array}{l}\text { Intervalo de } \\
\text { confiança de 95\% }\end{array}$ \\
\hline $\begin{array}{l}\text { Escore de Constant-Murley (CM) } \\
\text { x escore Modified-University of } \\
\text { California at Los Angeles } \\
\text { Shoulder Rating Scale (UCLA-M)* }\end{array}$ & $0,000^{1}$ & $-0,875--0,468$ \\
\hline Sexo x escore CM** & $0,525^{2}$ & $-9,1-14,5$ \\
\hline Idade x tipo de síntese** & $0,116^{2}$ & $-20,4--1,6$ \\
\hline $\begin{array}{l}\text { Escore CM } x \text { tipo de síntese } \\
\text { para o sexo masculino** }\end{array}$ & $0,483^{2}$ & $-9,2-11,2$ \\
\hline $\begin{array}{l}\text { Escore CM } x \text { tipo de síntese } \\
\text { para o sexo feminino** }\end{array}$ & $0,502^{2}$ & $-4,1-3,6$ \\
\hline
\end{tabular}

Notas: *Teste de correlação de Spearman. ** Teste $t$ de Student. ${ }^{1}$ Estatisticamente significativo. ${ }^{2}$ Sem significância estatística.

traram uma maior incidência desse tipo de fratura (68\%), e o alto percentual de ATs pode explicar tal situação, uma vez que nesses casos há relação com a alta energia do trauma, segundo Stegeman et al. ${ }^{23} \mathrm{Em}$ um outro estudo, ${ }^{24}$ tal 
característica foi identificada como fator de risco para pseudoartrose e, até mesmo, associada a piores escores funcionais. Em nosso estudo, contudo, não houve diferença significativa na comparação entre os tipos de traços de fratura e os escores funcionais.

A maior crítica em relação ao tratamento cirúrgico deve-se à proeminência do material de síntese, pois a clavícula tem localização subcutânea. Similar fato foi encontrado em nosso estudo, no qual a maioria das queixas dos pacientes foi a de que a placa estava saliente (44,1\%), o que corrobora a técnica cirúrgica e os achados em outros estudos. Wang et $\mathrm{al}^{25}$ encontraram $40 \%$ de complicações relacionadas à placa proeminente ou aos parafusos, assim como Nourian et al, $^{10}$ que descreveram que a abordagem superior acarreta alta taxa de problemas com as placas e a necessidade de uma nova cirurgia para a retirada do implante.

No contexto da funcionalidade do ombro, Naveen et $a^{8}{ }^{8}$ ao término do seu seguimento, encontraram um escore CM de 94 nos pacientes tratados cirurgicamente, e resultados similares foram encontrados em nosso estudo. As médias dos escores CM e UCLA-M, em nosso estudo, foram de 91,59 e 31,29 , respectivamente. As metanálises de Woltz et al ${ }^{16} \mathrm{e}$ Smeeing et $\mathrm{al}^{2}$ compararam o tratamento cirúrgico com o conservador, e eles encontraram melhores resultados que favorecem o primeiro; entretanto a diferença não é clinicamente relevante, dado que é preciso uma diferença pelo menos 15 pontos para haver algum impacto funcional no escore CM.

Ademais, van der Ven Denise et $\mathrm{al}^{26}$ e Naveen et $\mathrm{al}^{8}$ constataram que o tratamento cirúrgico traz benefícios mais rápidos do que o tratamento conservador, como a imediata estabilização, analgesia, facilita a mobilização precoce e, por conseguinte, proporciona um retorno mais rápido ao trabalho. Porém, quando analisada a função do ombro, os dois grupos só apresentam diferença significativa no período inicial do tratamento, até seis semanas em geral. Com cerca de 24 semanas, a variação entre os escores torna-se sem significância clínica, e os escores se equivalem no longo prazo com ambos os tratamentos.

Contudo, a principal vantagem do tratamento cirúrgico é facilitar a redução da fratura e a diminuição da ocorrência de pseudoartrose. Essa complicação é a que piora a qualidade de vida e diminui a funcionalidade do ombro. Asadollahi et al $^{17}$ analisaram as complicações relacionadas à fixação por placa, e, em seu estudo, a pseudoartrose ocorreu em $2,7 \%$ dos casos, e uma taxa semelhante foi encontrada no presente estudo (2,9\%). Esses números estão bem abaixo dos encontrados na literatura atual em relação à opção não cirúrgica, e, segundo George et al, ${ }^{27}$ essa situação pode acometer de 15 a $26 \%$ dos adultos.

Há algumas limitações em nosso estudo. Primeiro, o número limitado de pacientes no estudo, e a dificuldade de entrar em contato com os pacientes, o que poderia evidenciar melhores resultados. Segundo, os pacientes não foram vistos no mesmo momento de evolução de suas fraturas, dado que este estudo tinha caráter retrospectivo, o que pode ter melhorado os escores funcionais.

\section{Conclusão}

Concluímos que o tratamento cirúrgico acarreta bons resultados funcionais após uma fratura diafiasária de clavícula, independente do tipo de traço de fratura, com baixa taxa de pseudoartrose. A complicação mais associada ao tratamento foi relacionada à proeminência do material de síntese. Portanto, tais resultados podem ser agregados à literatura disponível, para que o tratamento das fraturas de clavícula seja otimizado.

Conflito de interesses

Os autores declaram não haver conflito de interesses.

\section{Referências}

1 Gao Y, Chen W, Liu YJ, Li X, Wang HL, Chen ZY. Plating versus intramedullary fixation for mid-shaft clavicle fractures: a systemic review and meta-analysis. PeerJ 2016;4(01): e1540

2 Smeeing DPJ, van der Ven DJC, Hietbrink F, et al. Surgical versus nonsurgical treatment for midshaft clavicle fractures in patients aged 16 years and older: a systematic review, meta-analysis, and comparison of randomized controlled trials and observational studies. Am J Sports Med 2017;45(08):1937-1945

3 Napora JK, Grimberg D, Childs BR, Vallier HA. Factors affecting functional outcomes after clavicle fracture. JAm Acad Orthop Surg 2016;24(10):721-727

4 Burnham JM, Kim DC, Kamineni S. Midshaft clavicle fractures: a critical review. Orthopedics 2016;39(05):e814-e821

5 Ojeda-Reyes ÁJ, Barragán-Hervella GR, Vallecillo-Velázquez H, Alvarado-Ortega I, Romero-Figueroa MS, Montiel-Jarquín ÁJ. Evaluación funcional y radiológica de pacientes con fractura diafisiaria de clavícula manejados quirúrgicamente. Rev Med Inst Mex Seguro Soc 2016;54(12):254-258

6 Zhang B, Zhu Y, Zhang F, Chen W, Tian Y, Zhang Y. Meta-analysis of plate fixation versus intramedullary fixation for the treatment of mid-shaft clavicle fractures. Scand J Trauma Resusc Emerg Med 2015;23(01):27

7 Lenza M, Taniguchi LF, Ferretti M, Ferretti M. Figure-of-eight bandage versus arm sling for treating middle-third clavicle fractures in adults: study protocol for a randomised controlled trial. Trials 2016;17(01):229

8 Naveen BM, Joshi GR, Harikrishnan B. Management of mid-shaft clavicular fractures: comparison between non-operative treatment and plate fixation in 60 patients. Strateg Trauma Limb Reconstr 2017;12(01):11-18

9 Melean PA, Zuniga A, Marsalli M, et al. Surgical treatment of displaced middle-third clavicular fractures: a prospective, randomized trial in a working compensation population. JShoulder Elbow Surg 2015;24(04):587-592

10 Nourian A, Dhaliwal S, Vangala S, Vezeridis PS. Midshaft Fractures of the Clavicle: a meta-analysis comparing surgical fixation via anteriorinferior plating versus superior plating. J Orthop Trauma 2017;31(09):461-467

11 Donnelly TD, Macfarlane RJ, Nagy MT, Ralte P, Waseem M. Fractures of the clavicle: an overview. Open Orthop J 2013;7 (01):329-333

12 Naimark M, Dufka FL, Han R, et al. Plate fixation of midshaft clavicular fractures: patient-reported outcomes and hardwarerelated complications. JShoulder Elbow Surg 2016;25(05): 739-746

13 Barreto RP, Barbosa ML, Balbinotti MA, Mothes FC, da Rosa LH, Silva MF. The Brazilian version of the Constant-Murley Score (CMS-BR): convergent and construct validity, internal consistency, and unidimensionality. Rev Bras Ortop 2016;51(05):515-520 
14 Oku EC, Andrade AP, Stadiniky SP. Tradução e adaptação cultural do modified-University of California at Los Angeles Shoulder Rating Scale para a Língua Portuguesa. Translation and Cultural Adaptation of the Modified-University of California at Los Angeles Shoulder Rating Scale to Portuguese language. Rev Bras Reumatol 2006;46(04):246-252

15 Habermeyer P, Magosch P, Lichtenberg S. Classifications and Scores of the Shoulder. Berlin: Springer; 2006

16 Woltz S, Krijnen P, Schipper IB. Plate fixation versus nonoperative treatment for displaced midshaft clavicular fractures. J Bone Joint Surg Am 2017;99(12):1051-1057

17 Asadollahi S, Hau RC, Page RS, Richardson M, Edwards ER. Complications associated with operative fixation of acute midshaft clavicle fractures. Injury 2016;47(06):1248-1252

18 Devji T, Kleinlugtenbelt Y, Evaniew N, Ristevski B, Khoudigian S, Bhandari M. Operative versus nonoperative interventions for common fractures of the clavicle: a meta-analysis of randomized controlled trials. CMAJ Open 2015;3(04):E396-E405

19 Kihlström C, Möller M, Lönn K, Wolf O. Clavicle fractures: epidemiology, classification and treatment of 2422 fractures in the Swedish Fracture Register; an observational study. BMC Musculoskelet Disord 2017;18(01):82

20 Robinson CM, Goudie EB, Murray IR, et al. Open reduction and plate fixation versus nonoperative treatment for displaced midshaft clavicular fractures: a multicenter, randomized, controlled trial. J Bone Joint Surg Am 2013;95(17):1576-1584
21 Murray IR, Foster CJ, Eros A, Robinson CM. Risk factors for nonunion after nonoperative treatment of displaced midshaft fractures of the clavicle. JBone Joint Surg Am 2013;95(13):1153-1158

22 Ban I, Nowak J, Virtanen K, Troelsen A. Overtreatment of displaced midshaft clavicle fractures. Acta Orthop 2016;87(06):541-545

23 Stegeman SA, Roeloffs CWJ, van den Bremer J, Krijnen P, Schipper IB. The relationship between trauma mechanism, fracture type, and treatment of midshaft clavicular fractures. Eur J Emerg Med 2013;20(04):268-272

24 Liu W, Xiao J, Ji F, Xie Y, Hao Y. Intrinsic and extrinsic risk factors for nonunion after nonoperative treatment of midshaft clavicle fractures. Orthop Traumatol Surg Res 2015;101(02):197-200

25 Wang XH, Cheng L, Guo WJ, et al. Plate versus intramedullary fixation care of displaced midshaft clavicular fractures: a metaanalysis of prospective randomized controlled trials. Medicine (Baltimore) 2015;94(41):e1792

26 van der Ven Denise JC, Timmers TK, Flikweert PE, Van Ijseldijk ALA, van Olden GD. Plate fixation versus conservative treatment of displaced midshaft clavicle fractures: Functional outcome and patients' satisfaction during a mean follow-up of 5 years. Injury 2015;46(11):2223-2229

27 George DM, McKay BP, Jaarsma RL. The long-term outcome of displaced mid-third clavicle fractures on scapular and shoulder function: variations between immediate surgery, delayed surgery, and nonsurgical management. J Shoulder Elbow Surg 2015; 24(05):669-676 